\title{
Intussusception in a child with COVID-19 in the USA
}

\author{
Eseosa Amy Bazuaye-Ekwuyasi ${ }^{1}$ - Alvin C. Camacho ${ }^{1}$ (D) - Florentino Saenz Rios ${ }^{2} \cdot$ Andrew Torck $^{2}$. \\ Woongsoon John Choi ${ }^{1} \cdot$ Ebelosele E. Aigbivbalu ${ }^{3} \cdot$ Mohammed Q. Mehdi ${ }^{3} \cdot$ Kyle J. Shelton ${ }^{3} \cdot$ \\ Geetha L. Radhakrishnan ${ }^{3} \cdot$ Ravi S. Radhakrishnan ${ }^{4} \cdot$ Leonard E. Swischuk $^{1}$
}

Received: 17 August 2020 / Accepted: 27 September 2020 / Published online: 6 October 2020

(C) American Society of Emergency Radiology 2020

\begin{abstract}
COVID-19, caused by the novel coronavirus strain SARS-CoV-2 that emerged in late 2019, has resulted in a global pandemic. COVID-19 was initially believed to occur less frequently in children with relatively mild disease. However, severe disease and varied presentations have been reported in infected children, one of such being intussusception. There have only been three reported cases of intussusception in the pediatric population infected with COVID-19. In this paper, we will discuss the management and treatment of a novel fourth case of COVID-19-associated intussusception. This case is the first reported in the USA and suggests that COVID-19 may be implicated in the development of intussusception. Pediatricians should consider the possibility of intussusception when a child with COVID-19 presents with abdominal pain.
\end{abstract}

Keywords Pediatrics · Intussusception $\cdot$ COVID-19 $\cdot$ Coronavirus $\cdot$ SARS-CoV-2

$\begin{array}{ll}\begin{array}{l}\text { Abbreviations } \\ \text { SARS-CoV-2 }\end{array} & \begin{array}{l}\text { Severe acute respiratory syndrome } \\ \text { coronavirus 2 }\end{array} \\ \text { COVID-19 } & \begin{array}{l}\text { Coronavirus 2019 } \\ \text { Gastrointestinal }\end{array} \\ \text { GI } & \text { Polymerase chain reaction } \\ \text { PCR } & \text { Abdominal radiograph } \\ \text { AXR } & \text { World Health Organization } \\ \text { WHO } & \text { Multisystem inflammatory } \\ \text { MIS-C } & \text { syndrome in children } \\ \text { CDC } & \text { Centers for Disease Control } \\ \text { Tmax } & \text { Maximum temperature }\end{array}$

Alvin C. Camacho accamach@UTMB.EDU

1 Department of Radiology, University of Texas Medical Branch, 301 University Blvd, Galveston, TX 77555, USA

2 School of Medicine, University of Texas Medical Branch, 301 University Blvd, Galveston, TX 77555, USA

3 Department of Pediatrics, University of Texas Medical Branch, 301 University Blvd, Galveston, TX 77555, USA

4 Department of Surgery, University of Texas Medical Branch, 301 University Blvd, Galveston, TX 77555, USA

\section{What's new?}

There have been three reported cases of intussusception associated with COVID-19 worldwide [1-3] with ours being the fourth case and the first in the USA.

\section{Introduction}

The severe acute respiratory syndrome coronavirus 2 (SARSCoV-2) virus first appeared in late 2019 and is responsible for the global coronavirus 2019 (COVID-19) pandemic. Unlike in the adult population where respiratory symptoms are characteristic, few infected children are symptomatic with only about half presenting with fever or cough. Additionally, gastrointestinal (GI) symptoms have been documented to occur more commonly in children than in adults. Intussusception is a possible but rare GI manifestation of COVID-19 infection with only three other reported cases worldwide [1-3], making this case the first of its kind to be reported in the USA.

\section{Case presentation}

A 9-month-old Hispanic male initially presented to the outpatient clinic on June 27, 2020, with complaints of increased fussiness, congestion, cough, and sneezing for 4 days and 


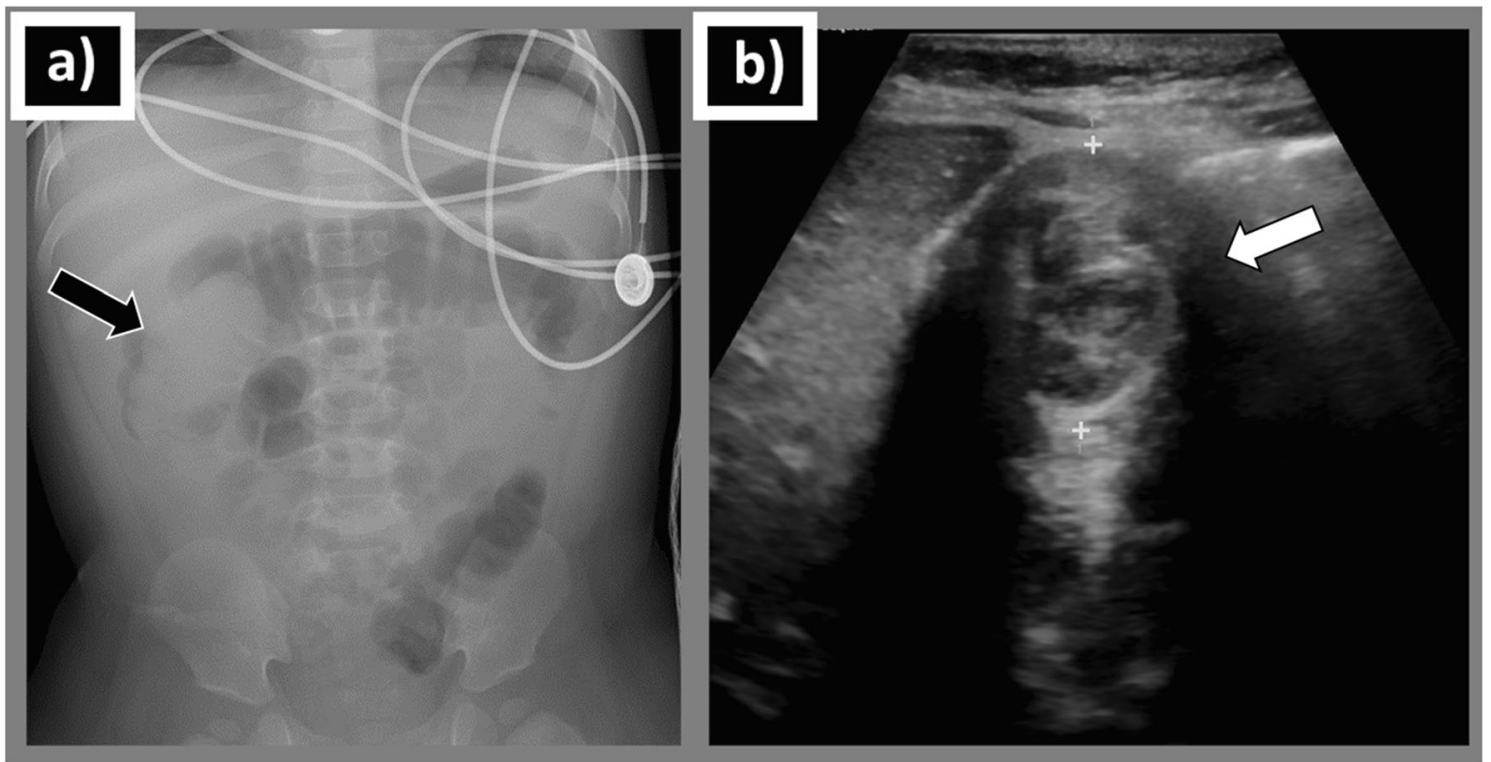

Fig. 1 a Frontal view abdominal radiograph shows an elongated filling defect (arrow) within the ascending colon and hepatic flexure with an airfilled transverse colon distally, consistent with the "colon cutoff sign" of intussusception. b Transverse gray-scale abdominal sonogram shows concentric alternating echogenic and hypoechoic bands (arrow), consistent with the target sign of intussusception. No lead points including enlarged lymph nodes were identified on sonographic examination
Fig. 2 Hydrostatic reduction of intussusception using

Gastrografin contrast solution. a Initial fluoroscopic image shows an ileocolic intussusception as an intracolonic filling defect at the level of the hepatic flexure (arrow). The distal colon is opacified by contrast up to the level of the intussusceptum. b, c Progressive unfolding of the telescoped bowel (arrows). d Complete reduction of the previously noted intussusception with opacification of the entire colon and retrograde filling of the ileum
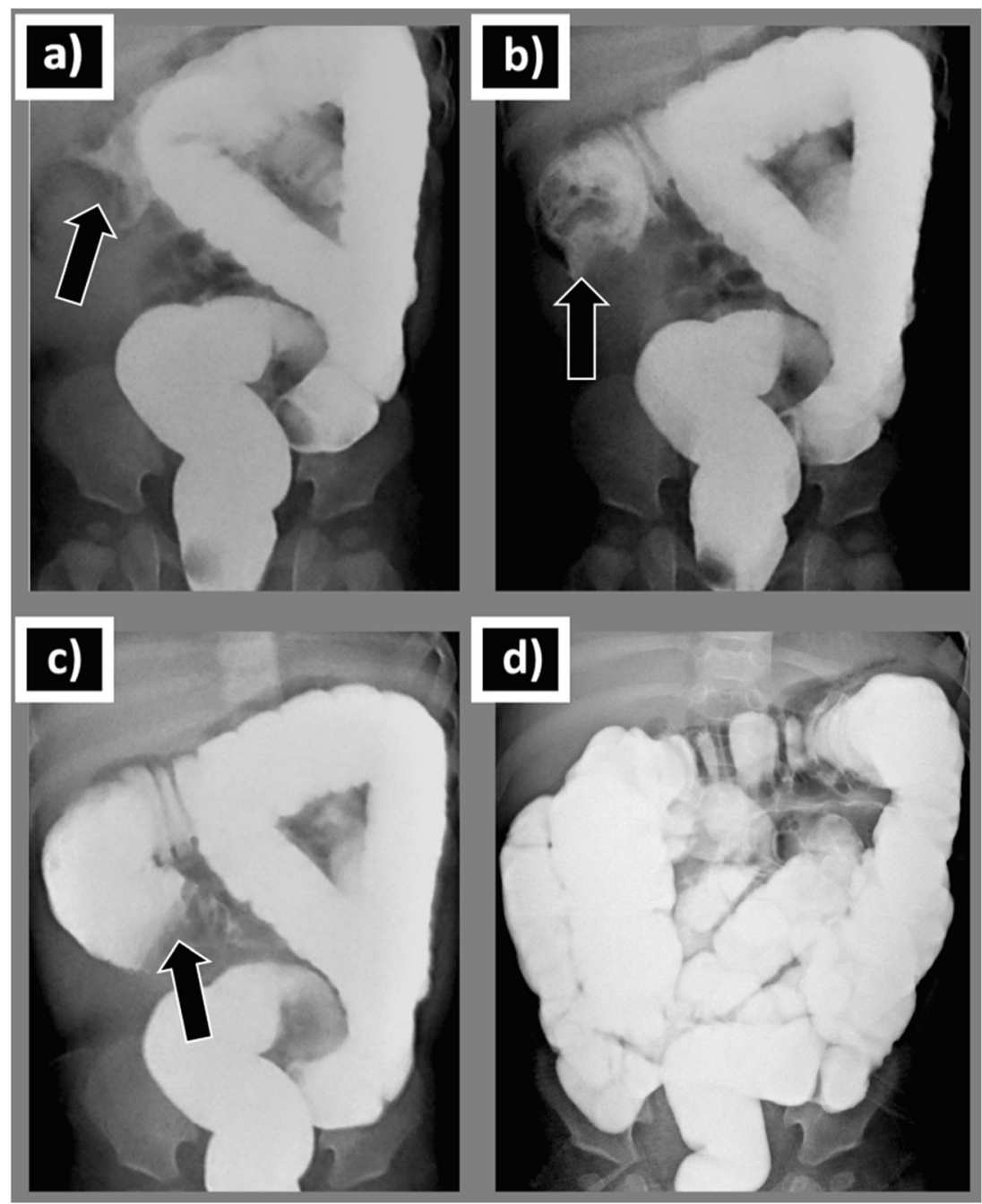
Table 1 Criteria for multi-system inflammatory syndrome in children (MIS-C) associated with COVID-19 (based on the CDC case definition $^{14}$ )

Must meet all of the following criteria:

\begin{tabular}{ll}
\hline 1 & Age $<21$ years old \\
2 & Fever, either $\geq 38{ }^{\circ} \mathrm{C}$ or subjective fever, for $\geq 24 \mathrm{~h}$ \\
3 & Laboratory signs of inflammation including any of \\
the following: \\
a. Lymphopenia \\
b. Mild anemia \\
c. Thrombocytopenia \\
d. Elevated ESR \\
e. Elevated CRP \\
f. Elevated procalcitonin \\
g. Elevated fibrinogen \\
h. Elevated lactic acid dehydrogenase \\
i. Elevated ferritin \\
j. Hypoalbuminemia \\
k. Transaminitis \\
Multi-system involvement including at least two of \\
the following: \\
a. Gastrointestinal (e.g., nausea, vomiting, diarrhea) \\
b. Renal (e.g., elevated creatinine, proteinuria) \\
c. Cardiac (e.g., elevated pro-BNP, elevated troponin, \\
positive echocardiographic is findings) \\
d. Mucocutaneous inflammation (e.g., palms, soles of \\
feet, conjunctiva, oral mucosa) \\
e. Coagulopathy (e.g., elevated PT, aPTT, D-dimer) \\
f. Hypotension/shock \\
No alternative diagnosis or explanation for signs of \\
inflammation \\
Current or recent positive COVID-19 with at least \\
one of the following: \\
a. PCR \\
b. Serology \\
c. Antigen \\
d. Unprotected COVID-19 exposure within the last 4 weeks \\
6
\end{tabular}

fever of $100.7^{\circ} \mathrm{F}\left(38.2^{\circ} \mathrm{C}\right)$ for 1 day. The patient's contact history was positive for similar symptoms in close relatives. SARS-CoV-2 polymerase chain reaction (PCR) molecular testing was performed which yielded a positive result.

Two days following the initial outpatient visit, the patient presented to the emergency room with complaints of vomiting, episodic abdominal pain, decreased oral intake, and blood streaked stool. Signs of dehydration were noted on physical examination, but the abdominal exam was unremarkable. Urinalysis revealed ketonuria $(80 \mathrm{mg} / \mathrm{dl})$ and proteinuria $(30 \mathrm{mg} / \mathrm{dl})$. Complete blood count was significant for decreased lymphocytes at $1.6 \times 10^{3} \mu \mathrm{l}$. Abdominal radiograph (AXR) and abdominal ultrasound confirmed the presence of ileocolic intussusception (Fig. 1).

Non-surgical management with fluoroscopy-guided hydrostatic reduction of the intussusception was performed using a solution of saline and Gastrografin $®$ (diatrizoate meglumine and diatrizoate sodium solution) contrast material. Successful reduction was achieved in a single attempt without complications (Fig. 2). The patient remained stable and was discharged home the following day.

\section{Discussion}

COVID-19 refers to the infection caused by the novel 2019 coronavirus strain, SARS-CoV-2, that emerged in late 2019 and has been subsequently declared a global pandemic by the World Health Organization (WHO) on March 2, 2020. As of July 11,2020 , the virus has resulted in more than 12 million infections and over half a million deaths worldwide. The USA accounts for a quarter of these infections with more than 3 million cases and over 130,000 deaths [4]. Initially, COVID19 was believed to be less frequent and not as virulent in

Table 2 Comparison of presentation among SARS-CoV-2 intussusception cases

\begin{tabular}{|c|c|c|c|c|}
\hline Presentation & Bazuaye-Ekwuyasi et al. & Martinez-Castano et al. [3] & Moazzam et al. [1] & Cai et al. [2] \\
\hline Age in months & 9 & 6 & 4 & 10 \\
\hline Sex & Male & Male & Male & Female \\
\hline Exposure history & Yes; via relatives & Yes; via relatives & No & No \\
\hline Duration of symptoms/days & 6 & 2 & 2 & 1.25 \\
\hline Fever & Febrile, Tmax $-38.2^{\circ} \mathrm{C}$ & No fever & No fever & Febrile, Tmax $-39.8^{\circ} \mathrm{C}$ \\
\hline Prior URI symptoms & Yes, 2 days prior to presentation & No & $\begin{array}{l}\text { Yes, } 1 \text { week prior to } \\
\text { presentation }\end{array}$ & No \\
\hline Abdominal symptoms & $\begin{array}{l}\text { Episodic abdominal pain, } \\
\text { with darkened stools }\end{array}$ & $\begin{array}{l}\text { Vomiting, currant } \\
\text { jelly stools }\end{array}$ & $\begin{array}{l}\text { Episodic abdominal pain, } \\
\text { currant jelly stools }\end{array}$ & Vomiting, currant jelly stools \\
\hline Palpable sausage shaped mass & No & No & Yes & Not specified \\
\hline Type of intussusception & Ileocolic & Ileocecal & Ileocecal & Not specified \\
\hline Reduction of intussusception & Hydrostatic Reduction & Hydrostatic Reduction & Pneumatic Reduction & Pneumatic Reduction \\
\hline Outcome & Recovered & Recovered & Recovered & $\begin{array}{l}\text { Deceased-multiorgan } \\
\text { dysfunction syndrome }\end{array}$ \\
\hline
\end{tabular}

Tmax maximum temperature 
children. However, reports from Europe and the USA have emerged depicting an association between COVID-19 infection in children and a condition called multisystem inflammatory syndrome in children (MIS-C) [5]. The Center for Disease Control's (CDC) case definition of this syndrome is outlined in Table 1.

Intussusception is the most common abdominal emergency in young children [6] and the most common cause of intestinal obstruction in children between 3 months and 6 years [7] . There have been three reported cases of intussusception associated with COVID-19 worldwide [1-3] with ours being the fourth case and the first in the USA. A comparison of our case with the other documented cases is included in Table 2. Most pediatric intussusceptions are idiopathic with a precipitating lesion detected in only about $10-25 \%$ of cases $[6,8]$. Nonetheless, several factors have been implicated in its pathophysiology. Viral infections are the most prevalent etiologic factor [9] with the most commonly implicated virus being non-enteric adenoviruses [9]. In the limited cases with identifiable lead points, lymphoid hyperplasia of Peyer's Patches within the ileum was the most common instigating lead point [8]. Although the pathogenesis of COVID-19- related intussusception is not fully understood, it can be inferred to parallel the other viral associated intussusception cases. This involves an alteration in the peristaltic movement of the bowel wall which enables the telescoping of adjacent loops of bowel into each other, manifesting as intussusception [6]. Notably, the widespread immune activation that has been shown to be prevalent in COVID-19 may result in hypertrophy of the intestinal Peyer's patches thereby inciting intussusception.

Of the four reported cases of COVID-19-related intussusception, there has been one mortality. This contrasts with the mortality rate of less than $1 \%$ in intussusception associated with other viruses. The heightened mortality in COVID-19associated intussusception is dependent on the association with MIS-C as seen in the case in Wuhan, China [2] who developed multiple organ failure and eventually died. Hence, prompt testing, institution of appropriate precautions, and preparation for possible complications are of vital importance in patients with COVID-19-related intussusception. Our case, however, did not meet the diagnostic criteria for MIS-C as outlined in Table 1.

The gold standard for management of intussusception in children is non-surgical reduction by hydrostatic or pneumatic enema under fluoroscopic or sonographic guidance with surgery reserved for complicated cases or after failure of nonsurgical reduction. All reported cases of COVID-19associated intussusception had successful non-surgical reduction, even the patient who eventually died. Hence, enema reduction should be attempted in uncomplicated cases of COVID-19-associated intussusception, as prescribed by current guidelines.

\section{Conclusion}

Our case report suggests that COVID-19 may be implicated in the development of intussusception. Hence, it is important that pediatricians consider the possibility of intussusception when a COVID-19 patient presents with abdominal pain. Also, as children infected with SARS-CoV-2 are more likely to be asymptomatic or exhibit non-respiratory symptoms, we propose that children with intussusception during this pandemic should be tested for SARS-CoV-2. This is imperative as timely diagnosis is crucial for the successful management of COVID-19 and avoidance of potentially severe complications, including MIS-C.

\section{Compliance with ethical standards}

Conflict of interest The authors declare that they have no conflict of interest.

\section{References}

1. Moazzam Z, Salim A, Ashraf A, Jehan F, Arshad M (2020) Intussusception in an infant as a manifestation of COVID-19. J Pediatr Surg Case Rep 59:101533-101533. https://doi.org/10.1016/ j.epsc.2020.101533

2. Cai X, Ma Y, Li S, Chen Y, Rong Z, Li W (2020) Clinical characteristics of 5 COVID-19 cases with non-respiratory symptoms as the first manifestation in children. Front Pediatr 8:258. https://doi.org/10. 3389/fped.2020.00258

3. Martínez-Castaño I, Calabuig-Barbero E, Gonzálvez-Piñera J, López-Ayala JM (2020) COVID-19 infection is a diagnostic challenge in infants with ileocecal intussusception. Pediatr Emerg Care 6:e368

4. CDC. Coronavirus disease 2019 (COVID-19) cases and deaths in the US. Accessed July 11th, 2020, https://www.cdc.gov/coronavirus/ 2019-ncov/cases-updates/us-cases-deaths.html

5. CDC. Multisystem inflammatory syndrome in children (MIS-C) associated with coronavirus disease 2019 (COVID-19). https:// emergency.cdc.gov/han/2020/han00432.asp

6. Cera SM (2008) Intestinal intussusception. Clin Colon Rectal Surg 21(2):106-113. https://doi.org/10.1055/s-2008-1075859

7. Waseem M, Rosenberg HK (2008) Intussusception. Pediatr Emerg Care 24(11):793-800. https://doi.org/10.1097/PEC. 0b013e31818c2a3e

8. Ntoulia A, Tharakan SJ, Reid JR, Mahboubi S (2016) Failed intussusception reduction in children: correlation between radiologic, surgical, and pathologic findings. AJR Am J Roentgenol 207(2):424433. https://doi.org/10.2214/ajr.15.15659

9. Okimoto S, Hyodo S, Yamamoto M, Nakamura K, Kobayashi M (2011) Association of viral isolates from stool samples with intussusception in children. Int J Infect Dis 15(9):e641-e645. https://doi. org/10.1016/j.ijid.2011.05.008

Publisher's note Springer Nature remains neutral with regard to jurisdictional claims in published maps and institutional affiliations. 